\title{
Efficient Attribute-Based Signatures for Non-monotone Predicates in the Standard Model
}

\author{
Tatsuaki Okamoto ${ }^{1}$ and Katsuyuki Takashima ${ }^{2}$ \\ ${ }^{1}$ NTT, 3-9-11 Midori-cho, Musashino-shi, Tokyo, 180-8585, Japan \\ okamoto.tatsuaki@lab.ntt.co.jp \\ ${ }^{2}$ Mitsubishi Electric, 5-1-1, Ofuna, Kamakura, Kanagawa, 247-8501, Japan \\ Takashima.Katsuyuki@aj.MitsubishiElectric.co.jp
}

\begin{abstract}
This paper presents a fully secure (adaptive-predicate unforgeable and private) attribute-based signature (ABS) scheme in the standard model. The security of the proposed ABS scheme is proven under standard assumptions, the decisional linear (DLIN) assumption and the existence of collision resistant (CR) hash functions. The admissible predicates of the proposed ABS scheme are more general than those of the existing ABS schemes, i.e., the proposed ABS scheme is the first to support general non-monotone predicates, which can be expressed using NOT gates as well as AND, OR, and Threshold gates, while the existing ABS schemes only support monotone predicates. The proposed ABS scheme is efficient and practical. Its efficiency is comparable to (several times worse than) that of the most efficient (almost optimally efficient) ABS scheme the security for which is proven in the generic group model.
\end{abstract}

\section{Introduction}

\subsection{Background}

The concept of digital signatures was introduced in the seminal paper by Diffie and Hellman in 1976. In this concept, a pair comprising a secret signing key, sk, and public verification key, pk, is generated for a signer, and signature $\sigma$ of message $m$ generated using sk is verified by the corresponding pk. Hence, the signer of $(m, \sigma)$ using sk is identified through pk. Although it is one of the requirements of signatures, there is no flexibility or privacy in the relationship between signers and claims attested by signatures due to the tight relation between sk and pk.

Recently, versatile and privacy-enhanced variants of digital signatures have been studied, where the relation between a signing key and verification key is more flexible or sophisticated. In this class of signatures, the signing key and verification key are parameterized by attribute $\boldsymbol{x}$ and predicate $\boldsymbol{v}$, respectively, and signed message $(m, \sigma)$ generated by the signing key with parameter $\boldsymbol{x}, \mathbf{s k}_{\boldsymbol{x}}$, is correctly verified by public-key pk and parameter $\boldsymbol{v},(\mathrm{pk}, \boldsymbol{v})$, iff predicate $\boldsymbol{v}$ accepts attribute $\boldsymbol{x}$, i.e., $\boldsymbol{v}(\boldsymbol{x})$ holds. The privacy of signers in this class of signatures requires that a signature (for predicate $\boldsymbol{v}$ ) generated by sk (where $\boldsymbol{v}(\boldsymbol{x})$ holds) release no information regarding attribute $\boldsymbol{x}$ except that $\boldsymbol{v}(\boldsymbol{x})$ holds.

D. Catalano et al. (Eds.): PKC 2011, LNCS 6571, pp. 35 52, 2011.

(C) International Association for Cryptologic Research 2011 
When predicate $\boldsymbol{v}$ is the equality with parameter $v$ (i.e., $\boldsymbol{v}(x)$ holds iff $x=v$ ), the class of signatures for this predicate is identity-based signatures (IBS) [25]. Here note that there is no room for privacy in IBS, since predicate $\boldsymbol{v}$ uniquely identifies attribute $x$ of the signer's secret key, $\mathrm{sk}_{x}$, such that $x=v$.

Group signatures [9] are also in this class of signatures with another type of predicate $\boldsymbol{v}$, where $\boldsymbol{v}(\boldsymbol{x})$ holds iff predicate parameter $v$ is the group identity (or $\mathrm{pk}_{v}$ is a public key identifying group $v$ ) and attribute $x$ is a member identity of group $v$ (or $\mathrm{sk}_{x}$ is a secret key of member $x$ of group $v$ ). Due to the privacy requirement, signatures generated using $\mathbf{s k}_{x}$ release no information regarding member identity $x$ except that $x$ is a member of group $v$ (Note that the concept of group signatures traditionally requires the privacy-revocation property as well as the above-mentioned privacy).

Recently, this class of signatures with more sophisticated predicates, attributebased signatures (ABS), has been extensively studied 11 13, 16 19, 24, 27], where $\boldsymbol{x}$ for signing key $\mathbf{s k}_{\boldsymbol{x}}$ is a tuple of attributes $\left(x_{1}, \ldots, x_{i}\right)$, and $\boldsymbol{v}$ for verification is a threshold or access structure predicate. The widest class of predicates in the existing ABS schemes are monotone access structures [18, 19], where predicate $\boldsymbol{v}$ is specified by a monotone span program (MSP), $(M, \rho)$, along with a tuple of attributes $\left(v_{1}, \ldots, v_{j}\right)$, and $\boldsymbol{v}(\boldsymbol{x})$ holds iff $\operatorname{MSP}(M, \rho)$ accepts the truthvalue vector of $\left(\mathrm{T}\left(x_{i_{1}}=v_{1}\right), \ldots, \mathrm{T}\left(x_{i_{j}}=v_{j}\right)\right)$. Here, $\mathrm{T}(\psi):=1$ if $\psi$ is true, and $\mathrm{T}(\psi):=0$ if $\psi$ is false (For example, $\mathrm{T}(x=v):=1$ if $x=v$, and $\mathrm{T}(x=v):=0$ if $x \neq v$ ). In general, such a predicate can be expressed using AND, OR, and Threshold gates.

An example of such monotone predicate $\boldsymbol{v}$ for ABS is (Institute = Univ. A) AND $($ TH2 $(($ Department $=$ Biology $),($ Gender $=$ Female $),($ Age $=50$ 's $))$ OR $($ Position $=$ Professor $)$ ), where TH2 means the threshold gate with threshold value 2. Attribute $\boldsymbol{x}_{A}$ of Alice is ((Institute $:=$ Univ. A), (Department := Biology), (Position $:=$ Postdoc), (Age $:=30)$, (Gender $:=$ Female)), and attribute $\boldsymbol{x}_{B}$ of Bob is ((Institute $:=$ Univ. A), (Department $:=$ Mathematics), (Position $:=$ Professor), (Age $:=45)$ (Gender $:=$ Male))). Although their attributes, $\boldsymbol{x}_{A}$ and $\boldsymbol{x}_{B}$, are quite different, it is clear that $\boldsymbol{v}\left(\boldsymbol{x}_{A}\right)$ and $\boldsymbol{v}\left(\boldsymbol{x}_{B}\right)$ hold, and that there are many other attributes that satisfy $\boldsymbol{v}$. Hence Alice and Bob can generate a signature on this predicate, and due to the privacy requirement of ABS, a signature for $\boldsymbol{v}$ releases no information regarding the attribute or identity of the signer, i.e., Alice or Bob (or other), except that the attribute of the signer satisfies $\boldsymbol{v}$.

There are many applications of ABS such as attribute-based messaging (ABM), attribute-based authentication, trust-negotiation and leaking secrets (see [18, 19] for more details).

The security conditions for ABS are given hereafter (see Section 3.2 for the formal definitions).

Unforgeability: A valid signature should be produced only by a single signer whose attribute $\boldsymbol{x}$ satisfies the claimed predicate $\boldsymbol{v}$, not by a collusion of users who pooled their attributes together. More formally, no poly-time adversary can produce a valid signature for a pair comprising predicate and message 
$(\boldsymbol{v}, m)$, even if the adversary adaptively chooses $(\boldsymbol{v}, m)$ after executing secretkey and signing oracle attacks, provided that $\boldsymbol{x}$ where $\boldsymbol{v}(\boldsymbol{x})$ holds is not queried to the secret-key oracle and $(\boldsymbol{v}, m)$ is not queried to the signing oracle (We simply call this unforgeability "adaptive-predicate unforgeability" or more simply "unforgeability").

We can also define a weaker class of unforgeability, 'selective-predicate unforgeability,' where an adversary should choose predicate $\boldsymbol{v}$ for the forgery signature before executing secret-key and signing oracle attacks.

Privacy: A signature for predicate $\boldsymbol{v}$ generated using secret key $\mathbf{s k}_{\boldsymbol{x}}$ releases no information regarding attribute $\boldsymbol{x}$ except that $\boldsymbol{v}(\boldsymbol{x})$ holds.

More formally, for any pair of attributes $\left(\boldsymbol{x}_{1}, \boldsymbol{x}_{2}\right)$, predicate $\boldsymbol{v}$ and message $m$, for which $\boldsymbol{v}\left(\boldsymbol{x}_{1}\right)$ and $\boldsymbol{v}\left(\boldsymbol{x}_{2}\right)$ hold simultaneously, the distributions of two valid signatures $\sigma\left(m, \boldsymbol{v}, \mathbf{s k}_{\boldsymbol{x}_{1}}\right)$ and $\sigma\left(m, \boldsymbol{v}, \mathbf{s k}_{\boldsymbol{x}_{2}}\right)$ are equivalent, where $\sigma\left(m, \boldsymbol{v}, \mathbf{s k}_{\boldsymbol{x}}\right)$ is a correctly generated signature for $(m, \boldsymbol{v})$ using correct secret key $\mathbf{s k}_{\boldsymbol{x}}$ with attribute $\boldsymbol{x}$ (We simply call this condition "privacy").

Full Security: We say that an ABS scheme is fully secure if it satisfies adaptivepredicate unforgeability and privacy.

Maji, Prabhakaran, and Rosulek [18, 19] presented ABS schemes for the widest class of predicates among the existing ABS schemes, monotone access structure predicates, which cover threshold predicates as special cases. The scheme shown in [18] is an almost optimally efficient ABS scheme, but the security was only proven in the generic group model. The scheme shown in [19] is the only existing ABS scheme for which (full) security was proven in the standard model. It is, however, much less efficient and more complicated than the scheme in [18] since it employs the Groth-Sahai NIZK protocols [10] as building blocks.

Li, Au, Susilo, Xie and Ren [16], Li and Kim [17], and Shahandashti and Safavi-Naini 24] presented ABS schemes that are proven to be secure in the standard model. However, the proven security is not the full security, but a weaker level of security with selective-predicate unforgeability. Moreover, the admissible predicates in 17] are limited to conjunction or $(n, n)$-threshold predicates, and those of [16, 24] are limited to $(k, n)$-threshold predicates.

Guo and Zeng [11] and Yang, Cao and Dong [27] presented ABS schemes for threshold predicates, but their security definitions do not include the privacy condition of ABS.

Khader [12, 13] presented ABS schemes for monotone access structure predicates. These schemes, however, do not satisfy the privacy condition of ABS, since they only conceal the identity of the signer. They also reveal the attributes that the signer used to generate the signature. In addition, the security is proven in a non-standard model, the random oracle model.

Based on this background, there are two major problems in the existing ABS schemes.

1. No ABS scheme for non-monotone predicates, which can be expressed using NOT gates as well as AND, OR and Threshold gates, has been proposed (even in a weaker security notion or a non-standard model). 
2. The only fully secure ABS scheme in the standard model [19] is much less efficient than the (almost optimally efficient) ABS scheme in the generic group model [18].

Non-monotone predicates should be used in many ABS applications. For example, annual review reports in the Mathematics Department of University A are submitted by reviewers, and these reports are anonymously signed by the reviewers through ABS with some predicates. The predicates may be selected freely by them (signers) except that it should be in the following form: NOT((Institute = Univ. A) AND (Department = Mathematics)) AND ( ...).

\subsection{Our Results}

This paper addresses these problems simultaneously.

- This paper proposes the first fully secure (i.e., adaptive-predicate unforgeable and perfectly private) ABS scheme for a wide class of predicates, nonmonotone access structures, where $\boldsymbol{x}$ for signing key $\mathbf{s k}_{\boldsymbol{x}}$ is a tuple of attributes $\left(x_{1}, \ldots, x_{i}\right)$, non-monotone predicate $\boldsymbol{v}$ is specified by a span pro$\operatorname{gram}(\mathrm{SP})(M, \rho)$ along with a tuple of attributes $\left(v_{1}, \ldots, v_{j}\right)$, and $\boldsymbol{v}(\boldsymbol{x})$ holds iff $\mathrm{SP}(M, \rho)$ accepts the truth-value vector of $\left(\mathrm{T}\left(x_{i_{1}}=v_{1}\right), \ldots, \mathrm{T}\left(x_{i_{j}}=v_{j}\right)\right)$. Our scheme can be generalized using non-monotone access structures combined with inner-product relations (see Definition 5] and the remark). More precisely, attribute $\boldsymbol{x}$ for signing key $\mathbf{s k}_{\boldsymbol{x}}$ is a tuple of attribute vectors (e.g., $\left.\left(\vec{x}_{1}, \ldots, \vec{x}_{i}\right) \in \mathbb{F}_{q}^{n_{1}+\cdots+n_{i}}\right)$, and predicate $\boldsymbol{v}$ for verification is a nonmonotone access structure or span program $(\mathrm{SP})(M, \rho)$ along with a tuple of attribute vectors (e.g., $\left.\left(\vec{v}_{1}, \ldots, \vec{v}_{j}\right) \in \mathbb{F}_{q}^{n_{1}+\cdots+n_{j}}\right)$, where the componentwise inner-product relations for attribute vectors (e.g., $\left\{\vec{x}_{i_{\iota}} \cdot \vec{v}_{\iota}=0\right.$ or not \}$_{\iota \in\{1, \ldots, j\})}$ are input to SP $(M, \rho)$. Namely, $\boldsymbol{v}(\boldsymbol{x})$ holds iff the truth-value vector of $\left(\mathrm{T}\left(\vec{x}_{i_{1}} \cdot \vec{v}_{1}=0\right), \ldots, \mathrm{T}\left(\vec{x}_{i_{j}} \cdot \vec{v}_{j}=0\right)\right)$ is accepted by $\operatorname{SP}(M, \rho)$.

Remark: In our scheme (Section 4), attribute $\boldsymbol{x}$ is expressed by the form $\Gamma:=\left\{\left(t, x_{t}\right) \mid t \in T \subseteq\{1, \ldots, d\}\right\}$ in place of just an attribute tuple $\left(x_{1}, \ldots, x_{i}\right)$, where $t$ identifies a sub-universe or category of attributes, and $x_{t}$ is an attribute in sub-universe $t$ (examples of $\left(t, x_{t}\right)$ are (Name, Alice) and (Age, 38)). Predicate $\boldsymbol{v}$ is expressed by $\mathbb{S}:=(M, \rho)$, where $\rho$ is abused as $\rho$ (defined by SP) combined with $\left\{\left(t_{i}, v_{i}\right) \mid i=1, \ldots, \ell\right\}$ (see Definitions 4 and 5 for the difference regarding $\rho$ in $\mathrm{SP}$ and $\mathbb{S}$ ).

- The proposed ABS scheme is proven to be fully secure under standard assumptions, the decisional linear (DLIN) assumption (over prime order pairing groups) and the existence of collision resistant (CR) hash functions, in the standard model.

- In contrast to the ABS scheme in [19] that employs the Groth-Sahai NIZK protocols, our ABS scheme is more directly constructed without using any general subprotocols like NIZK. Our construction is based on the dual pairing vector spaces (DPVS) proposed by Okamoto and Takashima [14, 20 22], which can be realized from any type of (e.g., symmetric or asymmetric) 
prime order bilinear pairing groups. See Section 2.1 for the concept and actual construction of DPVS.

- To prove the security (especially the unforgeability), this paper employs the techniques for fully secure functional encryption (FE) 14, 22], which elaborately combine the dual system encryption methodology proposed by Waters [26] and DPVS.

Note that although the techniques for the FE schemes in [14, 22] can be employed for ABS, it is still a challenging task to construct a fully secure ABS scheme, since the security requirements of ABS and FE differ in some important points, for example, the privacy condition is required in ABS but there is no counterpart notion in FE. This paper develops several novel techniques for our ABS scheme. See Section 4.1 for more details.

- The efficiency of the proposed ABS scheme is comparable to that of the most efficient ABS scheme in the generic group model [18], and better than that of the only existing fully secure ABS scheme in the standard model [19]. See Section 4.4 for a comparison.

- This paper also presents an extension, multi-authority (MA) setting, of the proposed ABS scheme in Section 5. One of the merits of our MA-ABS scheme is that it is seamlessly extended from the original (single-authority (SA)) setting, in which the signing and verification algorithms of the MA-ABS scheme are essentially the same as those of the original ABS (SA-ABS) scheme.

In MA-ABS, each authority called an attribute authority is responsible for a category of attributes, and a user obtains a part of secret key for each attribute from an attribute authority responsible for the category of the attribute. We follow the model of MA-ABS introduced in [18, 19], where a central trustee in addition to attribute authorities is required but no interaction among attribute authorities (and the trustee) is necessary, and different attribute authorities may not trust each other, nor even be aware of each other.

We prove that the proposed MA-ABS scheme is fully secure (in the sense of the MA-ABS model of [18, 19]) under the DLIN assumption and CR hash functions in the standard model (see the full version of this paper for the proof). Our MA-ABS scheme is almost as efficient as the original SA-ABS scheme.

\subsection{Related Works}

- Ring and mesh signatures: Ring and mesh signatures [4, 23] are related to ABS.

In the ring signatures, the claimed predicate on a signature of message $m$ is that $m$ is endorsed by one of the users identified by the list of public keys $\left(\mathrm{pk}_{1}, \mathrm{pk}_{2}, \ldots\right)$, or the predicate is a disjunction of a list of public keys. A valid ring signature can be generated by one of the listed users.

The mesh signatures are an extension of ring signatures, where the predicate is an access structure on a list of pairs comprising a message and public key $\left(m_{i}, \mathrm{pk}_{i}\right)$, and a valid mesh signature can be generated by a person who has enough standard signatures $\sigma_{i}$ on $m_{i}$, each valid under $\mathrm{pk}_{i}$, to satisfy the given access structure. 
A crucial difference between mesh signatures and ABS is the security against the collusion of users. In mesh signatures, several users can collude by pooling their signatures together and create signatures that none of them could produce individually. That is, such collusion is considered to be legitimate in mesh signatures. In contrast, the security against collusion attacks is one of the basic requirements in ABS and MA-ABS, as described in Section 1.1 and Section 5 .

- Anonymous credentials (ACs): Another related concept is ACs [2, 3, 58]. The notion of ACs also provides a functionality for users to demonstrate anonymously possession of attributes, but the goals of ACs and ABS differ in several points.

As mentioned in [19], ACs and ABS aim at different goals: ACs target very strong anonymity even in the registration phase, whereas under less demanding anonymity requirements in the registration phase, ABS aims to achieve more expressive functionalities, more efficient constructions and new applications. In addition, ABS is a signature scheme and a simpler primitive compared with ACs.

\subsection{Notations}

When $A$ is a random variable or distribution, $y \stackrel{\mathrm{R}}{\leftarrow} A$ denotes that $y$ is randomly selected from $A$ according to its distribution. When $A$ is a set, $y \stackrel{U}{\longleftarrow} A$ denotes that $y$ is uniformly selected from $A . y:=z$ denotes that $y$ is set, defined or substituted by $z$. When $a$ is a fixed value, $A(x) \rightarrow a$ (e.g., $A(x) \rightarrow 1$ ) denotes the event that machine (algorithm) $A$ outputs $a$ on input $x$. A function $f: \mathbb{N} \rightarrow \mathbb{R}$ is negligible in $\lambda$, if for every constant $c>0$, there exists an integer $n$ such that $f(\lambda)<\lambda^{-c}$ for all $\lambda>n$.

We denote the finite field of order $q$ by $\mathbb{F}_{q}$, and $\mathbb{F}_{q} \backslash\{0\}$ by $\mathbb{F}_{q}^{\times}$. A vector symbol denotes a vector representation over $\mathbb{F}_{q}$, e.g., $\vec{x}$ denotes $\left(x_{1}, \ldots, x_{n}\right) \in \mathbb{F}_{q}^{n}$. For two vectors $\vec{x}=\left(x_{1}, \ldots, x_{n}\right)$ and $\vec{v}=\left(v_{1}, \ldots, v_{n}\right), \vec{x} \cdot \vec{v}$ denotes the inner-product $\sum_{i=1}^{n} x_{i} v_{i}$. The vector $\overrightarrow{0}$ is abused as the zero vector in $\mathbb{F}_{q}^{n}$ for any $n . X^{\mathrm{T}}$ denotes the transpose of matrix $X$. A bold face letter denotes an element of vector space $\mathbb{V}$, e.g., $\boldsymbol{x} \in \mathbb{V}$. When $\boldsymbol{b}_{i} \in \mathbb{V}(i=1, \ldots, n)$, $\operatorname{span}\left\langle\boldsymbol{b}_{1}, \ldots, \boldsymbol{b}_{n}\right\rangle \subseteq \mathbb{V}$ (resp. $\left.\operatorname{span}\left\langle\vec{x}_{1}, \ldots, \vec{x}_{n}\right\rangle\right)$ denotes the subspace generated by $\boldsymbol{b}_{1}, \ldots, \boldsymbol{b}_{n}\left(\right.$ resp. $\left.\vec{x}_{1}, \ldots, \vec{x}_{n}\right)$. For bases $\mathbb{B}:=\left(\boldsymbol{b}_{1}, \ldots, \boldsymbol{b}_{N}\right)$ and $\mathbb{B}^{*}:=$ $\left(\boldsymbol{b}_{1}^{*}, \ldots, \boldsymbol{b}_{N}^{*}\right),\left(x_{1}, \ldots, x_{N}\right)_{\mathbb{B}}:=\sum_{i=1}^{N} x_{i} \boldsymbol{b}_{i}$ and $\left(y_{1}, \ldots, y_{N}\right)_{\mathbb{B}^{*}}:=\sum_{i=1}^{N} y_{i} \boldsymbol{b}_{i}^{*}$.

\section{Preliminaries}

\subsection{Dual Pairing Vector Spaces by Direct Product of Symmetric Pairing Groups}

Definition 1. "Symmetric bilinear pairing groups" $\left(q, \mathbb{G}, \mathbb{G}_{T}, G, e\right)$ are a tuple of a prime $q$, cyclic additive group $\mathbb{G}$ and multiplicative group $\mathbb{G}_{T}$ of order $q$, $G \neq 0 \in \mathbb{G}$, and a polynomial-time computable nondegenerate bilinear pairing $e: \mathbb{G} \times \mathbb{G} \rightarrow \mathbb{G}_{T}$ i.e., $e(s G, t G)=e(G, G)^{\text {st }}$ and $e(G, G) \neq 1$. 
Let $\mathcal{G}_{\mathrm{bpg}}$ be an algorithm that takes input $1^{\lambda}$ and outputs a description of bilinear pairing groups $\left(q, \mathbb{G}, \mathbb{G}_{T}, G, e\right)$ with security parameter $\lambda$.

In this paper, we concentrate on the symmetric version of dual pairing vector spaces [14, 20 22] constructed by using symmetric bilinear pairing groups given in Definition 1 .

Definition 2. "Dual pairing vector spaces (DPVS)" $\left(q, \mathbb{V}, \mathbb{G}_{T}, \mathbb{A}, e\right)$ by a direct product of symmetric pairing groups $\left(q, \mathbb{G}, \mathbb{G}_{T}, G, e\right)$ are a tuple of prime $q, N$ dimensional vector space $\mathbb{V}:=\overbrace{\mathbb{G} \times \cdots \times \mathbb{G}}^{N}$ over $\mathbb{F}_{q}$, cyclic group $\mathbb{G}_{T}$ of order $q$, canonical basis $\mathbb{A}:=\left(\boldsymbol{a}_{1}, \ldots, \boldsymbol{a}_{N}\right)$ of $\mathbb{V}$, where $\boldsymbol{a}_{i}:=(\overbrace{0, \ldots, 0}^{i-1}, G, \overbrace{0, \ldots, 0}^{N-i})$, and pairing $e: \mathbb{V} \times \mathbb{V} \rightarrow \mathbb{G}_{T}$.

The pairing is defined by $e(\boldsymbol{x}, \boldsymbol{y}):=\prod_{i=1}^{N} e\left(G_{i}, H_{i}\right) \in \mathbb{G}_{T}$ where $\boldsymbol{x}:=\left(G_{1}, \ldots\right.$, $\left.G_{N}\right) \in \mathbb{V}$ and $\boldsymbol{y}:=\left(H_{1}, \ldots, H_{N}\right) \in \mathbb{V}$. This is nondegenerate bilinear i.e., $e(s \boldsymbol{x}, t \boldsymbol{y})=e(\boldsymbol{x}, \boldsymbol{y})^{s t}$ and if $e(\boldsymbol{x}, \boldsymbol{y})=1$ for all $\boldsymbol{y} \in \mathbb{V}$, then $\boldsymbol{x}=\mathbf{0}$. For all $i$ and $j, e\left(\boldsymbol{a}_{i}, \boldsymbol{a}_{j}\right)=e(G, G)^{\delta_{i, j}}$ where $\delta_{i, j}=1$ if $i=j$, and 0 otherwise, and $e(G, G) \neq 1 \in \mathbb{G}_{T}$.

DPVS also has linear transformations $\phi_{i, j}$ on $\mathbb{V}$ s.t. $\phi_{i, j}\left(\boldsymbol{a}_{j}\right)=\boldsymbol{a}_{i}$ and $\phi_{i, j}\left(\boldsymbol{a}_{k}\right)=$ $\mathbf{0}$ if $k \neq j$, which can be easily achieved by $\phi_{i, j}(\boldsymbol{x}):=(\overbrace{0, \ldots, 0}^{i-1}, G_{j}, \overbrace{0, \ldots, 0}^{N-i})$ where $\boldsymbol{x}:=\left(G_{1}, \ldots, G_{N}\right)$. We call $\phi_{i, j}$ "canonical maps".

DPVS generation algorithm $\mathcal{G}_{\mathrm{dpvs}}$ takes input $1^{\lambda}(\lambda \in \mathbb{N})$ and $N \in \mathbb{N}$, and outputs a description of param $_{\mathbb{V}}:=\left(q, \mathbb{V}, \mathbb{G}_{T}, \mathbb{A}, e\right)$ with security parameter $\lambda$ and $N$-dimensional $\mathbb{V}$. It can be constructed by using $\mathcal{G}_{\mathrm{bpg}}$.

The asymmetric version of DPVS, $\left(q, \mathbb{V}, \mathbb{V}^{*}, \mathbb{G}_{T}, \mathbb{A}, \mathbb{A}^{*}, e\right)$, is given in the full version of [22]. The above symmetric version is obtained by identifying $\mathbb{V}=\mathbb{V}^{*}$ and $\mathbb{A}=\mathbb{A}^{*}$ in the asymmetric version. (For another construction of DPVS using higher genus Jacobians, see [20].)

\subsection{Decisional Linear (DLIN) Assumption}

Definition 3 (DLIN Assumption). The DLIN problem is to guess $\beta \in\{0,1\}$, given $\left(\operatorname{param}_{\mathbb{G}}, G, \xi G, \kappa G, \delta \xi G, \sigma \kappa G, Y_{\beta}\right) \stackrel{\mathrm{R}}{\leftarrow} \mathcal{G}_{\beta}^{\mathrm{DLIN}}\left(1^{\lambda}\right)$, where

$$
\begin{gathered}
\mathcal{G}_{\beta}^{\text {DLIN }}\left(1^{\lambda}\right): \operatorname{param}_{\mathbb{G}}:=\left(q, \mathbb{G}, \mathbb{G}_{T}, G, e\right) \stackrel{\mathrm{R}}{\leftarrow} \mathcal{G}_{\mathrm{bpg}}\left(1^{\lambda}\right), \\
\kappa, \delta, \xi, \sigma \stackrel{\mathrm{U}}{\leftarrow} \mathbb{F}_{q}, \quad Y_{0}:=(\delta+\sigma) G, \quad Y_{1} \stackrel{\cup}{\leftarrow}, \\
\quad \text { return }\left(\operatorname{param}_{\mathbb{G}}, G, \xi G, \kappa G, \delta \xi G, \sigma \kappa G, Y_{\beta}\right),
\end{gathered}
$$

for $\beta \longleftarrow\{0,1\}$. For a probabilistic machine $\mathcal{E}$, we define the advantage of $\mathcal{E}$ for the DLIN problem as: $\operatorname{Adv}_{\mathcal{E}}^{\mathrm{DLIN}}(\lambda):=\mid \operatorname{Pr}\left[\mathcal{E}\left(1^{\lambda}, \varrho\right) \rightarrow 1 \mid \varrho \stackrel{\mathrm{R}}{\leftarrow} \mathcal{G}_{0}^{\mathrm{DLIN}}\left(1^{\lambda}\right)\right]-$ $\operatorname{Pr}\left[\mathcal{E}\left(1^{\lambda}, \varrho\right) \rightarrow 1 \mid \varrho \stackrel{\mathrm{R}}{\leftarrow} \mathcal{G}_{1}^{\mathrm{DLIN}}\left(1^{\lambda}\right)\right] \mid$. The DLIN assumption is: For any probabilistic polynomial-time adversary $\mathcal{E}$, the advantage $\operatorname{Adv}_{\mathcal{E}}^{\mathrm{DLIN}}(\lambda)$ is negligible in $\lambda$. 


\subsection{Collision Resistant (CR) Hash Functions}

Let $\lambda \in \mathbb{N}$ be a security parameter. A collision resistant (CR) hash function family, $\mathrm{H}$, associated with $\mathcal{G}_{\text {bpg }}$ and a polynomial, $\operatorname{poly}(\cdot)$, specifies two items:

- A family of key spaces indexed by $\lambda$. Each such key space is a probability space on bit strings denoted by $\mathrm{KH}_{\lambda}$. There must exist a probabilistic polynomial-time algorithm whose output distribution on input $1^{\lambda}$ is equal to $\mathrm{KH}_{\lambda}$.

- A family of hash functions indexed by $\lambda$, hk $\stackrel{R}{\leftarrow} \mathrm{KH}_{\lambda}$ and $\mathrm{D}:=\{0,1\}^{\text {poly }(\lambda)}$. Each such hash function $\mathrm{H}_{\mathrm{hk}}^{\lambda, \mathrm{D}}$ maps an element of $\mathrm{D}$ to an element of $\mathbb{F}_{q}^{\times}$ with $q$ that is the first element of output param $\mathbb{G}_{\mathbb{G}}$ of $\mathcal{G}_{\mathrm{bpg}}\left(1^{\lambda}\right)$. There must exist a deterministic polynomial-time algorithm that on input $1^{\lambda}$, hk and $\varrho \in \mathrm{D}$, outputs $\mathrm{H}_{\mathrm{hk}}^{\lambda, \mathrm{D}}(\varrho)$.

Let $\mathcal{E}$ be a probabilistic polynomial-time machine. For all $\lambda$, we define $\operatorname{Adv}_{\mathcal{E}}^{\mathrm{H}, \mathrm{CR}}(\lambda):=\operatorname{Pr}\left[\left(\varrho_{1}, \varrho_{2}\right) \in \mathrm{D}^{2} \wedge \varrho_{1} \neq \varrho_{2} \wedge \mathrm{H}_{\mathrm{hk}}^{\lambda, \mathrm{D}}\left(\varrho_{1}\right)=\mathrm{H}_{\mathrm{hk}}^{\lambda, \mathrm{D}}\left(\varrho_{2}\right)\right]$, where $\mathrm{D}:=\{0,1\}^{\text {poly }(\lambda)}$, hk $\stackrel{\mathrm{R}}{\leftarrow} \mathrm{KH}_{\lambda}$, and $\left(\varrho_{1}, \varrho_{2}\right) \stackrel{\mathrm{R}}{\leftarrow} \mathcal{E}\left(1^{\lambda}\right.$, hk, D). $\mathrm{H}$ is a collision resistant (CR) hash function family if for any probabilistic polynomial-time adversary $\mathcal{E}, \operatorname{Adv}_{\mathcal{E}}^{\mathrm{H}, \mathrm{CR}}(\lambda)$ is negligible in $\lambda$.

\section{ABS for Non-monotone Predicates}

\subsection{Span Programs and Non-monotone Access Structures}

Definition 4 (Span Programs [1]). Let $\left\{p_{1}, \ldots, p_{n}\right\}$ be a set of variables. A span program over $\mathbb{F}_{q}$ is a labeled matrix, $\hat{M}:=(M, \rho)$, where $M$ is a $(\ell \times r) m a-$ trix over $\mathbb{F}_{q}$ and $\rho$ is a labeling of the rows of $M$ by literals from $\left\{p_{1}, \ldots, p_{n}, \neg p_{1}, \ldots\right.$, $\left.\neg p_{n}\right\}$ (every row is labeled by one literal), i.e., $\rho:\{1, \ldots, \ell\} \rightarrow\left\{p_{1}, \ldots, p_{n}, \neg p_{1}\right.$, $\left.\ldots, \neg p_{n}\right\}$.

A span program accepts or rejects an input by the following criterion. For every input sequence $\delta \in\{0,1\}^{n}$ define submatrix $M_{\delta}$ of $M$ consisting of those rows whose labels are set to 1 by the input $\delta$, i.e., either rows labeled by some $p_{i}$ such that $\delta_{i}=1$ or rows labeled by some by some $\neg p_{i}$ such that $\delta_{i}=0$. (i.e., $\gamma:\{1, \ldots, \ell\} \rightarrow\{0,1\}$ is defined by $\gamma(j)=1$ if $\left[\rho(j)=p_{i}\right] \wedge\left[\delta_{i}=1\right]$ or $\left[\rho(j)=\neg p_{i}\right] \wedge\left[\delta_{i}=0\right]$, and $\gamma(j)=0$ otherwise. $M_{\delta}:=\left(M_{j}\right)_{\gamma(j)=1}$, where $M_{j}$ is the $j$-th row of $M$.)

Span program $\hat{M}$ accepts $\delta$ if and only if $\overrightarrow{1} \in \operatorname{span}\left\langle M_{\delta}\right\rangle$, i.e., some linear combination of the rows of $M_{\delta}$ gives the all one vector, $\overrightarrow{1}$. (The row vector has the value 1 in each coordinate.) A span program computes boolean function $f$ if it accepts exactly those inputs $\delta$ where $f(\delta)=1$.

A span program is called monotone if the labels of the rows are only the positive literals $\left\{p_{1}, \ldots, p_{n}\right\}$. Monotone span programs compute monotone functions. (So, a span program in general is "non"-monotone.)

We assume that access structure matrix $M$ (of type $\ell \times r$ ) satisfies the condition: $M_{i} \neq \overrightarrow{0}$ for $i=1, \ldots, \ell$. 
We now introduce a non-monotone access structure with evaluating map $\gamma$ by using the inner-product of attribute vectors in a general form. Although we will show the notion, security definition and security proof of the proposed ABS scheme in this general form, we will describe the proposed ABS scheme in a simpler form in Section 4.2. We will show this simpler form of Definition 5 in the remark.

Definition 5 (Inner-Products of Attribute Vectors and Access Structures $). \mathcal{U}_{t}\left(t=1, \ldots, d\right.$ and $\left.\mathcal{U}_{t} \subset\{0,1\}^{*}\right)$ is a sub-universe, a set of attributes, each of which is expressed by a pair of sub-universe $i d$ and $n_{t}$-dimensional vector, i.e., $(t, \vec{v})$, where $t \in\{1, \ldots, d\}$ and $\vec{v} \in \mathbb{F}_{q}^{n_{t}} \backslash\{\overrightarrow{0}\}$.

We now define such an attribute to be a variable, $p$, of span program $\hat{M}:=$ $(M, \rho)$ i.e., $p:=(t, \vec{v})$. Access structure $\mathbb{S}$ is span program $\hat{M}:=(M, \rho)$ along with variables $p:=(t, \vec{v}), p^{\prime}:=\left(t^{\prime}, \vec{v}^{\prime}\right), \ldots$, i.e., $\mathbb{S}:=(M, \rho)$ such that $\rho$ : $\{1, \ldots, \ell\} \rightarrow\left\{(t, \vec{v}),\left(t^{\prime}, \vec{v}^{\prime}\right), \ldots, \neg(t, \vec{v}), \neg\left(t^{\prime}, \vec{v}^{\prime}\right), \ldots\right\}$.

Let $\Gamma$ be a set of attributes, i.e., $\Gamma:=\left\{\left(t, \vec{x}_{t}\right) \mid \vec{x}_{t} \in \mathbb{F}_{q}^{n_{t}} \backslash\{\overrightarrow{0}\}, 1 \leq t \leq d\right\}$.

When $\Gamma$ is given to access structure $\mathbb{S}, \operatorname{map} \gamma:\{1, \ldots, \ell\} \rightarrow\{0,1\}$ for span program $\hat{M}:=(M, \rho)$ is defined as follows: For $i=1, \ldots, \ell$, set $\gamma(i)=1$ if $\left[\rho(i)=\left(t, \vec{v}_{i}\right)\right] \wedge\left[\left(t, \vec{x}_{t}\right) \in \Gamma\right] \wedge\left[\vec{v}_{i} \cdot \vec{x}_{t}=0\right]$ or $\left[\rho(i)=\neg\left(t, \vec{v}_{i}\right)\right] \wedge\left[\left(t, \vec{x}_{t}\right) \in \Gamma\right]$ $\wedge\left[\vec{v}_{i} \cdot \vec{x}_{t} \neq 0\right]$. Set $\gamma(i)=0$ otherwise.

Access structure $\mathbb{S}:=(M, \rho)$ accepts $\Gamma$ iff $\overrightarrow{1} \in \operatorname{span}\left\langle\left(M_{i}\right)_{\gamma(i)=1}\right\rangle$.

Remark: A simpler form of the inner-product relations in the above-mentioned access structures is a special case when $n_{t}=2$ for all $t \in\{1, \ldots, d\}$, and $\vec{x}:=$ $(1, x)$ and $\vec{v}:=(v,-1)$. Hence, $\left(t, \vec{x}_{t}\right):=\left(t,\left(1, x_{t}\right)\right)$ and $\left(t, \vec{v}_{i}\right):=\left(t,\left(v_{i},-1\right)\right)$, but we often denote them shortly by $\left(t, x_{t}\right)$ and $\left(t, v_{i}\right)$. Then, $\mathbb{S}:=(M, \rho)$ such that $\rho:\{1, \ldots, \ell\} \rightarrow\left\{(t, v),\left(t^{\prime}, v^{\prime}\right), \ldots \neg(t, v), \neg\left(t^{\prime}, v^{\prime}\right), \ldots\right\}\left(v, v^{\prime}, \ldots \in \mathbb{F}_{q}\right)$, and $\Gamma:=\left\{\left(t, x_{t}\right) \mid x_{t} \in \mathbb{F}_{q}, 1 \leq t \leq d\right\}$.

When $\Gamma$ is given to access structure $\mathbb{S}$, map $\gamma:\{1, \ldots, \ell\} \rightarrow\{0,1\}$ for span program $\hat{M}:=(M, \rho)$ is defined as follows: For $i=1, \ldots, \ell$, set $\gamma(i)=1$ if $[\rho(i)=$ $\left.\left(t, v_{i}\right)\right] \wedge\left[\left(t, x_{t}\right) \in \Gamma\right] \wedge\left[v_{i}=x_{t}\right]$ or $\left[\rho(i)=\neg\left(t, v_{i}\right)\right] \wedge\left[\left(t, x_{t}\right) \in \Gamma\right] \wedge\left[v_{i} \neq x_{t}\right]$. Set $\gamma(i)=0$ otherwise.

We now construct a secret-sharing scheme for a (non-monotone) access structure (span program).

Definition 6. A secret-sharing scheme for access structure $\mathbb{S}:=(M, \rho)$ is:

1. Let $M$ be an $\ell \times r$ matrix, and column vector $\vec{f}^{\mathrm{T}}:=\left(f_{1}, \ldots, f_{r}\right)^{\mathrm{T}} \stackrel{\mathrm{U}}{\longleftarrow} \mathbb{F}_{q}^{r}$. Then, $s_{0}:=\overrightarrow{1} \cdot \vec{f}^{\mathrm{T}}=\sum_{k=1}^{r} f_{k}$ is the secret to be shared, and $\vec{s}^{\mathrm{T}}:=$ $\left(s_{1}, \ldots, s_{\ell}\right)^{\mathrm{T}}:=M \cdot \vec{f}^{\mathrm{T}}$ is the vector of $\ell$ shares of secret $s_{0}$ and share $s_{i}$ belongs to $\rho(i)$.

2. If access structure $\mathbb{S}:=(M, \rho)$ accepts $\Gamma$, i.e., $\overrightarrow{1} \in \operatorname{span}\left\langle\left(M_{i}\right)_{\gamma(i)=1}\right\rangle$ with $\gamma:\{1, \ldots, \ell\} \rightarrow\{0,1\}$, then there exist constants $\left\{\alpha_{i} \in \mathbb{F}_{q} \mid i \in I\right\}$ such that $I \subseteq\{i \in\{1, \ldots, \ell\} \mid \gamma(i)=1\}$ and $\sum_{i \in I} \alpha_{i} s_{i}=s_{0}$. Furthermore, these constants $\left\{\alpha_{i}\right\}$ can be computed in time polynomial in the size of matrix $M$. 


\subsection{Definitions and Security of ABS}

Definition 7 (Attribute-Based Signatures : ABS). An attribute-based signature scheme consists of four algorithms.

Setup This is a randomized algorithm that takes as input security parameter and format $\vec{n}:=\left(d ; n_{1}, \ldots, n_{d}\right)$ of attributes. It outputs public parameters pk and master key sk.

KeyGen This is a randomized algorithm that takes as input a set of attributes, $\Gamma:=\left\{\left(t, \vec{x}_{t}\right) \mid \vec{x}_{t} \in \mathbb{F}_{q}^{n_{t}} \backslash\{\overrightarrow{0}\}, 1 \leq t \leq d\right\}$, pk and sk. It outputs signature generation key $\mathrm{sk}_{\Gamma}$.

Sig This is a randomized algorithm that takes as input message $m$, access structure $\mathbb{S}:=(M, \rho)$, signature generation key $\mathrm{sk}_{\Gamma}$, and public parameters $\mathrm{pk}$ such that $\mathbb{S}$ accepts $\Gamma$. It outputs signature $\sigma$.

Ver This takes as input message $m$, access structure $\mathbb{S}$, signature $\sigma$ and public parameters pk. It outputs boolean value accept $:=1$ or reject $:=0$.

An ABS scheme should have the following correctness property: for all (sk, pk) $\stackrel{\mathrm{R}}{\leftarrow} \operatorname{Setup}\left(1^{\lambda}, \vec{n}\right)$, all messages $m$, all attribute sets $\Gamma$, all signing keys $\mathrm{sk}_{\Gamma} \stackrel{\mathrm{R}}{\longleftarrow}$ KeyGen $(\mathrm{pk}, \mathrm{sk}, \Gamma)$, all access structures $\mathbb{S}$ such that $\mathbb{S}$ accepts $\Gamma$, and all signatures $\sigma \stackrel{\mathrm{R}}{\leftarrow} \operatorname{Sig}\left(\mathrm{pk}, \mathrm{sk}_{\Gamma}, m, \mathbb{S}\right)$, it holds that $\operatorname{Ver}(\mathrm{pk}, m, \mathbb{S}, \sigma)=1$ with probability 1 .

Definition 8 (Perfect Privacy). An ABS scheme is perfectly private, if, for all (sk, pk) $\stackrel{\mathrm{R}}{\leftarrow} \operatorname{Setup}\left(1^{\lambda}, \vec{n}\right)$, all messages $m$, all attribute sets $\Gamma_{1}$ and $\Gamma_{2}$, all signing keys $\mathrm{sk}_{\Gamma_{1}} \stackrel{\mathrm{R}}{\leftarrow} \mathrm{KeyGen}\left(\mathrm{pk}, \mathrm{sk}, \Gamma_{1}\right)$ and $\mathrm{sk}_{\Gamma_{2}} \stackrel{\mathrm{R}}{\leftarrow} \mathrm{KeyGen}\left(\mathrm{pk}, \mathrm{sk}, \Gamma_{2}\right)$, all access structures $\mathbb{S}$ such that $\mathbb{S}$ accepts $\Gamma_{1}$ and $\mathbb{S}$ accepts $\Gamma_{2}$, distributions $\operatorname{Sig}\left(\mathrm{pk}, \mathrm{sk}_{\Gamma_{1}}, m\right.$, $\mathbb{S})$ and $\operatorname{Sig}\left(\mathrm{pk}^{\mathrm{sk}} \mathrm{\Gamma}_{2}, m, \mathbb{S}\right)$ are equal.

For an ABS scheme with prefect privacy, we define algorithm AltSig (pk, sk, $m, \mathbb{S}$ ) with $\mathbb{S}$ and master key sk instead of $\Gamma$ and $\mathrm{sk}_{\Gamma}$ : First, generate $\mathrm{sk}_{\Gamma} \stackrel{\mathrm{R}}{\leftarrow} \mathrm{KeyGen}$ (pk, sk, $\Gamma$ ) for arbitrary $\Gamma$ which satisfies $\mathbb{S}$, then $\sigma \stackrel{\mathrm{R}}{\leftarrow} \operatorname{Sig}\left(\mathrm{pk}, \mathrm{sk}_{\Gamma}, m, \mathbb{S}\right)$. return $\sigma$.

Since the correct distribution on signatures can be perfectly simulated without taking any private information as input, signatures must not leak any such private information of the signer.

Definition 9 (Unforgeability). For an adversary, $\mathcal{A}$, we define $\operatorname{Adv}_{\mathcal{A}}^{\mathrm{ABS}, \mathrm{UF}}(\lambda)$ to be the success probability in the following experiment for any security parameter $\lambda$. An ABS scheme is existentially unforgeable if the success probability of any polynomial-time adversary is negligible:

1. Run (sk, pk) $\stackrel{\mathrm{R}}{\leftarrow} \operatorname{Setup}\left(1^{\lambda}, \vec{n}\right)$ and give $\mathrm{pk}$ to the adversary.

2. The adversary is given access to oracles KeyGen $(\mathrm{pk}, \mathrm{sk}, \cdot)$ and $\mathrm{AltSig}(\mathrm{pk}, \mathrm{sk}, \cdot, \cdot)$.

3. At the end, the adversary outputs $\left(m^{\prime}, \mathbb{S}^{\prime}, \sigma^{\prime}\right)$.

We say the adversary succeeds if $\left(m^{\prime}, \mathbb{S}^{\prime}\right)$ was never queried to the AltSig oracle, $\mathbb{S}^{\prime}$ does not accept any $\Gamma$ queried to the KeyGen oracle, and $\operatorname{Ver}\left(\mathrm{pk}, m^{\prime}, \mathbb{S}^{\prime}, \sigma^{\prime}\right)=1$. 


\section{Proposed ABS Scheme}

\subsection{Construction Ideas}

Here, we will show some basic ideas to construct the proposed ABS scheme. Our ABS scheme is constructed on a ciphertext policy $(\mathrm{CP})$ functional encryption (FE) scheme 22], which is adaptively payload-hiding against chosen-plaintext attacks. The description of the CP-FE scheme is given in the full version of [22].

Roughly speaking, a secret signing key, $\mathrm{sk}_{\Gamma}$, with attribute set $\Gamma$ and a verification text, $\overrightarrow{\boldsymbol{c}}$, with access structure $\mathbb{S}$ (for signature verification) in our ABS scheme correspond to a secret decryption key, $\mathrm{sk}_{\Gamma}$, with $\Gamma$ and a ciphertext, $\overrightarrow{\boldsymbol{c}}$, with $\mathbb{S}$ in the CP-FE scheme, respectively. No counterpart of a signature, $\vec{s}^{*}$, in the ABS exists in the CP-FE, and the privacy property for signature $\vec{s}^{*}$ is also specific in ABS. Signature $\vec{s}^{*}$ in ABS may be interpreted to be a decryption key specialized to decrypt a ciphertext with access structure $\mathbb{S}$, that is delegated from secret key $\mathrm{sk}_{\Gamma}$.

The algorithms of the proposed ABS scheme can be described in the light of such correspondence to the CP-FE scheme:

Setup. Almost the same as that in the CP-FE scheme except that $\left\{\widehat{\mathbb{B}}_{t}^{*}\right\}_{t=1, \ldots, d+1}$ are revealed as a public parameter in our ABS, while they are secret in the CP-FE scheme. They are published in our ABS for the signature generation procedure Sig to meet the privacy of signers (for randomization). This implies an important gap between CP-FE and ABS.

KeyGen. Almost the same as that in the CP-FE scheme except that a (7 dimensional) space with basis $\mathbb{B}_{d+1}^{*}$ is additionally introduced in our ABS and two elements $\boldsymbol{k}_{d+1,1}^{*}$ and $\boldsymbol{k}_{d+1,2}^{*}$ in this space are included in a secret signing key in order to embed the hash value, $\mathbf{H}_{\mathrm{hk}}^{\lambda, \mathrm{D}}(m \| \mathbb{S})$, of message $m$ and access structure $\mathbb{S}$ in signature $\vec{s}^{*}$.

Sig. Specific in ABS. To meet the privacy condition for $\vec{s}^{*}$, a novel technique is employed to randomly generate a signature from $\mathrm{sk}_{\Gamma}$ and $\left\{\widehat{\mathbb{B}}_{t}^{*}\right\}_{t=1, \ldots, d+1}$.

Ver. Signature $\vec{s}^{*}$ in the ABS is an endorsement to message $m$ by a signer with attributes accepted by access structure $\mathbb{S}$. The signature verification in our ABS checks whether signature (or specific decryption key) $\vec{s}^{*}$ works as a decryption key to decrypt a verification text (or a ciphertext) associated with $\mathbb{S}$ and $\boldsymbol{H}_{\mathrm{hk}}^{\lambda, \mathrm{D}}(m \| \mathbb{S})$.

Security proofs. Roughly speaking, the adaptive-predicate unforgeability of the ABS under the KeyGen oracle attacks can be guaranteed by the adaptive payload-hiding property of the CP-FE, since a forged signature implies a decryption key specified for the challenge ciphertext to break the payloadhiding. Note that there are many subtleties in the proof of unforgeability for the ABS, e.g., the unforgeability should be ensured in the ABS even when publishing $\left\{\widehat{\mathbb{B}}_{t}^{*}\right\}_{t=1, \ldots, d+1}$ for the privacy requirement, while they are secret in the CP-FE. We develop a novel technique to resolve the difficulty. See the full version of this paper for more details. 


\subsection{Construction}

For simplicity, here, we describe our ABS scheme for a specific parameter $\vec{n}:=$ $(d ; 2, \ldots, 2)$ (see the remark of Definition 5). A general form of our ABS scheme is given in the full version.

We define function $\widetilde{\rho}:\{1, \ldots, \ell\} \rightarrow\{1, \ldots, d\}$ by $\widetilde{\rho}(i):=t$ if $\rho(i)=(t, v)$ or $\rho(i)=\neg(t, v)$, where $\rho$ is given in access structure $\mathbb{S}:=(M, \rho)$. In the proposed scheme, we assume that $\widetilde{\rho}$ is injective for $\mathbb{S}:=(M, \rho)$. The full version of this paper shows how to relax the restriction.

$$
\begin{aligned}
& \operatorname{Setup}\left(1^{\lambda}, \vec{n}:=(d ; 2, \ldots, 2)\right): \operatorname{param}_{\mathbb{G}}:=\left(q, \mathbb{G}, \mathbb{G}_{T}, G, e\right) \stackrel{R}{\leftarrow} \mathcal{G}_{\text {bpg }}\left(1^{\lambda}\right), \\
& \mathrm{hk} \stackrel{\mathrm{R}}{\leftarrow} \mathrm{KH}_{\lambda}, \psi \stackrel{\cup}{\leftarrow} \mathbb{F}_{q}^{\times}, N_{0}:=4, N_{t}:=7 \text { for } t=1, \ldots, d+1, \\
& \text { for } t=0, \ldots, d+1, \quad \operatorname{param}_{\mathbb{V}_{t}}:=\left(q, \mathbb{V}_{t}, \mathbb{G}_{T}, \mathbb{A}_{t}, e\right):=\mathcal{G}_{\mathrm{dpvs}}\left(1^{\lambda}, N_{t}, \operatorname{param}_{\mathbb{G}}\right) \text {, } \\
& X_{t}:=\left(\chi_{t, i, j}\right)_{i, j} \stackrel{\cup}{\leftarrow} G L\left(N_{t}, \mathbb{F}_{q}\right),\left(\vartheta_{t, i, j}\right)_{i, j}:=\psi \cdot\left(X_{t}^{-1}\right)^{\mathrm{T}}, \\
& \boldsymbol{b}_{t, i}:=\left(\chi_{t, i, 1}, \ldots, \chi_{t, i, N_{t}}\right)_{\mathbb{A}_{t}}, \mathbb{B}_{t}:=\left(\boldsymbol{b}_{t, 1}, \ldots, \boldsymbol{b}_{t, N_{t}}\right) \text {, } \\
& \boldsymbol{b}_{t, i}^{*}:=\left(\vartheta_{t, i, 1}, \ldots, \vartheta_{t, i, N_{t}}\right)_{\mathbb{A}_{t}}, \mathbb{B}_{t}^{*}:=\left(\boldsymbol{b}_{t, 1}^{*}, \ldots, \boldsymbol{b}_{t, N_{t}}^{*}\right), \\
& g_{T}:=e(G, G)^{\psi}, \quad \operatorname{param}_{\vec{n}}:=\left(\left\{\text { param }_{\mathbb{V}_{t}}\right\}_{t=0, \ldots, d+1}, g_{T}\right) \text {, } \\
& \widehat{\mathbb{B}}_{0}:=\left(\boldsymbol{b}_{0,1}, \boldsymbol{b}_{0,4}\right), \quad \widehat{\mathbb{B}}_{t}:=\left(\boldsymbol{b}_{t, 1}, \boldsymbol{b}_{t, 2}, \boldsymbol{b}_{t, 7}\right) \text { for } t=1, \ldots, d+1, \\
& \widehat{\mathbb{B}}_{t}^{*}:=\left(\boldsymbol{b}_{t, 1}^{*}, \boldsymbol{b}_{t, 2}^{*}, \boldsymbol{b}_{t, 5}^{*}, \boldsymbol{b}_{t, 6}^{*}\right) \text { for } t=1, \ldots, d+1, \\
& \text { sk }:=\boldsymbol{b}_{0,1}^{*}, \quad \text { pk }:=\left(1^{\lambda}, \text { hk, param } \vec{n},\left\{\widehat{\mathbb{B}}_{t}\right\}_{t=0, \ldots, d+1},\left\{\widehat{\mathbb{B}}_{t}^{*}\right\}_{t=1, \ldots, d+1}, \boldsymbol{b}_{0,3}^{*}\right) \text {. } \\
& \text { return sk, pk. } \\
& \operatorname{KeyGen}\left(\text { pk, sk, } \Gamma:=\left\{\left(t, x_{t}\right) \mid 1 \leq t \leq d\right\}\right) \text { : } \\
& \delta \longleftarrow \mathbb{F}_{q}^{\times}, \quad \varphi_{0}, \varphi_{t, \iota}, \varphi_{d+1,1, \iota}, \varphi_{d+1,2, \iota} \longleftarrow \mathbb{F}_{q} \text { for } t=1, \ldots, d ; \iota=1,2 ; \\
& \boldsymbol{k}_{0}^{*}:=\left(\delta, 0, \varphi_{0}, 0\right)_{\mathbb{B}_{0}^{*}} \\
& \boldsymbol{k}_{t}^{*}:=\left(\delta\left(1, x_{t}\right), 0,0, \varphi_{t, 1}, \varphi_{t, 2}, 0\right)_{\mathbb{B}_{t}^{*}} \text { for }\left(t, x_{t}\right) \in \Gamma, \\
& \boldsymbol{k}_{d+1,1}^{*}:=\left(\delta(1,0), 0,0, \varphi_{d+1,1,1}, \varphi_{d+1,1,2}, 0\right)_{\mathbb{B}_{d+1}^{*}}, \\
& \boldsymbol{k}_{d+1,2}^{*}:=\left(\delta(0,1), 0,0, \varphi_{d+1,2,1}, \varphi_{d+1,2,2}, \quad 0\right)_{\mathbb{B}_{d+1}^{*}}, \\
& T:=\{0,(d+1,1),(d+1,2)\} \cup\left\{t \mid 1 \leq t \leq d,\left(t, x_{t}\right) \in \Gamma\right\}, \\
& \text { return } \operatorname{sk}_{\Gamma}:=\left(\Gamma,\left\{\boldsymbol{k}_{t}^{*}\right\}_{t \in T}\right) \text {. }
\end{aligned}
$$

$\operatorname{Sig}\left(\mathrm{pk}, \mathrm{sk}_{\Gamma}, m, \mathbb{S}:=(M, \rho)\right):$ If $\mathbb{S}:=(M, \rho) \operatorname{accepts} \Gamma:=\left\{\left(t, x_{t}\right)\right\}$, then compute $I$ and $\left\{\alpha_{i}\right\}_{i \in I}$ such that $\sum_{i \in I} \alpha_{i} M_{i}=\overrightarrow{1}$, and

$$
\begin{gathered}
I \subseteq\left\{i \in\{1, \ldots, \ell\} \mid\left[\rho(i)=\left(t, v_{i}\right) \wedge\left(t, x_{t}\right) \in \Gamma \wedge v_{i}=x_{t}\right]\right. \\
\left.\vee\left[\rho(i)=\neg\left(t, v_{i}\right) \wedge\left(t, x_{t}\right) \in \Gamma \wedge v_{i} \neq x_{t}\right]\right\}, \\
\xi \leftarrow \mathbb{F}_{q}^{\times}, \quad\left(\beta_{i}\right) \longleftarrow\left\{\left(\beta_{1}, \ldots, \beta_{\ell}\right) \mid \sum_{i=1}^{\ell} \beta_{i} M_{i}=\overrightarrow{0}\right\}, \\
\boldsymbol{s}_{0}^{*}:=\xi \boldsymbol{k}_{0}^{*}+\boldsymbol{r}_{0}^{*}, \text { where } \boldsymbol{r}_{0}^{*} \longleftarrow \operatorname{span}\left\langle\boldsymbol{b}_{0,3}^{*}\right\rangle, \\
\boldsymbol{s}_{i}^{*}:=\gamma_{i} \cdot \xi \boldsymbol{k}_{t}^{*}+\sum_{\iota=1}^{2} y_{i, \iota} \cdot \boldsymbol{b}_{t, \iota}^{*}+\boldsymbol{r}_{i}^{*} \quad \text { for } 1 \leq i \leq \ell,
\end{gathered}
$$


where $\boldsymbol{r}_{i}^{*} \stackrel{U}{\leftarrow} \operatorname{span}\left\langle\boldsymbol{b}_{t, 5}^{*}, \boldsymbol{b}_{t, 6}^{*}\right\rangle$, and $\gamma_{i}, \vec{y}_{i}:=\left(y_{i, 1}, y_{i, 2}\right)$ are defined as if $i \in I \wedge \rho(i)=\left(t, v_{i}\right), \quad \gamma_{i}:=\alpha_{i}, \quad \vec{y}_{i}:=\beta_{i}\left(1, v_{i}\right)$,

if $i \in I \wedge \rho(i)=\neg\left(t, v_{i}\right), \quad \gamma_{i}:=\frac{\alpha_{i}}{v_{i}-x_{t}}, \quad \vec{y}_{i}:=\frac{\beta_{i}}{v_{i}-y_{i}}\left(1, y_{i}\right)$, where $y_{i} \stackrel{\cup}{\longleftarrow} \mathbb{F}_{q} \backslash\left\{v_{i}\right\}$,

if $i \notin I \wedge \rho(i)=\left(t, v_{i}\right), \quad \gamma_{i}:=0, \quad \vec{y}_{i}:=\beta_{i}\left(1, v_{i}\right)$,

if $i \notin I \wedge \rho(i)=\neg\left(t, v_{i}\right), \quad \gamma_{i}:=0, \quad \vec{y}_{i}:=\frac{\beta_{i}}{v_{i}-y_{i}}\left(1, y_{i}\right)$, where $y_{i} \stackrel{\cup}{\longleftarrow} \mathbb{F}_{q} \backslash\left\{v_{i}\right\}$,

$\boldsymbol{s}_{\ell+1}^{*}:=\xi\left(\boldsymbol{k}_{d+1,1}^{*}+\mathrm{H}_{\mathrm{hk}}^{\lambda, \mathrm{D}}(m \| \mathbb{S}) \cdot \boldsymbol{k}_{d+1,2}^{*}\right)+\boldsymbol{r}_{\ell+1}^{*}$, where $\boldsymbol{r}_{\ell+1}^{*} \longleftarrow \operatorname{U} \operatorname{span}\left\langle\boldsymbol{b}_{d+1,5}^{*}, \boldsymbol{b}_{d+1,6}^{*}\right\rangle$,

return $\vec{s}^{*}:=\left(s_{0}^{*}, \ldots, s_{\ell+1}^{*}\right)$.

$\operatorname{Ver}\left(\mathrm{pk}, m, \mathbb{S}:=(M, \rho), \overrightarrow{\boldsymbol{s}}^{*}\right): \vec{f} \longleftarrow \mathbb{F}_{q}^{r}, \vec{s}^{\mathrm{T}}:=\left(s_{1}, \ldots, s_{\ell}\right)^{\mathrm{T}}:=M \cdot \vec{f}^{\mathrm{T}}$, $s_{0}:=\overrightarrow{1} \cdot \vec{f}^{\mathrm{T}}, \eta_{0}, \eta_{\ell+1}, \theta_{\ell+1}, s_{\ell+1} \longleftarrow \mathbb{F}_{q}$,

$\boldsymbol{c}_{0}:=\left(-s_{0}-s_{\ell+1}, 0,0, \eta_{0}\right)_{\mathbb{B}_{0}}$,

for $1 \leq i \leq \ell$,

if $\rho(i)=\left(t, v_{i}\right)$, return 0 if $s_{i}^{*} \notin \mathbb{V}_{t}$, else $\boldsymbol{c}_{i}:=\left(s_{i}+\theta_{i} v_{i},-\theta_{i}, \quad 0,0,0,0, \quad \eta_{i}\right)_{\mathbb{B}_{t}}, \quad$ where $\theta_{i}, \eta_{i} \stackrel{\cup}{\leftarrow} \mathbb{F}_{q}$, if $\rho(i)=\neg\left(t, v_{i}\right)$, return 0 if $s_{i}^{*} \notin \mathbb{V}_{t}$, else $\boldsymbol{c}_{i}:=\left(s_{i}\left(v_{i},-1\right), 0,0,0,0, \eta_{i}\right)_{\mathbb{B}_{t}}, \quad$ where $\eta_{i} \longleftarrow \mathbb{F}_{q}$, $\boldsymbol{c}_{\ell+1}:=\left(s_{\ell+1}-\theta_{\ell+1} \cdot \mathrm{H}_{\mathrm{hk}}^{\lambda, \mathrm{D}}(m \| \mathbb{S}), \theta_{\ell+1}, \quad 0,0, \quad 0,0, \quad \eta_{\ell+1}\right)_{\mathbb{B}_{d+1}}$, return 0 if $e\left(\boldsymbol{b}_{0,1}, \boldsymbol{s}_{0}^{*}\right)=1$, return 1 if $\prod_{i=0}^{\ell+1} e\left(\boldsymbol{c}_{i}, \boldsymbol{s}_{i}^{*}\right)=1$, return 0 otherwise.

[Correctness] $\prod_{i=0}^{\ell+1} e\left(\boldsymbol{c}_{i}, \boldsymbol{s}_{i}^{*}\right)$

$=e\left(\boldsymbol{c}_{0}, \boldsymbol{k}_{0}^{*}\right)^{\xi} \cdot \prod_{i \in I} e\left(\boldsymbol{c}_{i}, \boldsymbol{k}_{t}^{*}\right)^{\gamma_{i} \xi} \cdot \prod_{i=1}^{\ell} \prod_{\iota=1}^{2} e\left(\boldsymbol{c}_{i}, \boldsymbol{b}_{t, \iota}^{*}\right)^{y_{i, \iota}} \cdot e\left(\boldsymbol{c}_{\ell+1}, \boldsymbol{s}_{\ell+1}^{*}\right)$

$=g_{T}^{\xi \delta\left(-s_{0}-s_{\ell+1}\right)} \cdot \prod_{i \in I} g_{T}^{\xi \delta \alpha_{i} s_{i}} \cdot \prod_{i=1}^{\ell} g_{T}^{\beta_{i} s_{i}} \cdot g_{T}^{\xi \delta s_{\ell+1}}$

$=g_{T}^{\xi \delta\left(-s_{0}-s_{\ell+1}\right)} \cdot g_{T}^{\xi \delta s_{0}} \cdot g_{T}^{\xi \delta s_{\ell+1}}=1$.

\subsection{Security}

Theorem 1. The proposed ABS scheme is perfectly private.

Theorem 2. The proposed ABS scheme is unforgeable (adaptive-predicate unforgeable) under the DLIN assumption and the existence of collision resistant hash functions.

For any adversary $\mathcal{A}$, there exist probabilistic machines $\mathcal{E}_{1}, \mathcal{E}_{2, h}^{+}, \mathcal{E}_{2, h+1} \quad(h=$ $\left.0, \ldots, \nu_{1}-1\right), \mathcal{E}_{3, h}, \mathcal{E}_{4, h} \quad\left(h=1, \ldots, \nu_{2}\right)$, whose running times are essentially the same as that of $\mathcal{A}$, such that for any security parameter $\lambda$, 


$$
\begin{aligned}
\operatorname{Adv}_{\mathcal{A}}^{\mathrm{ABS}, \mathrm{UF}}(\lambda) \leq \operatorname{Adv}_{\mathcal{E}_{1}}^{\mathrm{DLIN}}(\lambda)+\sum_{h=0}^{\nu_{1}-1}\left(\operatorname{Adv}_{\mathcal{E}_{2, h}^{+}}^{\mathrm{DLIN}}(\lambda)+\operatorname{Adv}_{\mathcal{E}_{2, h+1}}^{\mathrm{DLIN}}(\lambda)\right) \\
+\sum_{h=1}^{\nu_{2}}\left(\operatorname{Adv}_{\mathcal{E}_{3, h}}^{\mathrm{DLIN}}(\lambda)+\operatorname{Adv}_{\mathcal{E}_{4, h}}^{\mathrm{H}, \mathrm{CR}}(\lambda)\right)+\epsilon
\end{aligned}
$$

where $\nu_{1}$ is the maximum number of $\mathcal{A}$ 's KeyGen queries, $\nu_{2}$ is the maximum number of $\mathcal{A}$ 's AltSig queries, and $\epsilon:=\left((2 d+16) \nu_{1}+18 \nu_{2}+2 d+18\right) / q$.

The proofs of Theorems 1 and 2 are given in the full version of this paper.

\subsection{Performance}

In this section, we compare the efficiency and security of the proposed ABS scheme with the existing ABS schemes in the standard model (two typical instantiations) [19] as well as the ABS scheme in the generic group model [18] (as a benchmark). Since all of these schemes can be implemented over a prime order pairing group, the size of a group element can be around the size of $\mathbb{F}_{q}$ (e.g., 256 bits). In Table 1 l $\ell$ and $r$ represent the size of the underlying access structure matrix $M$ for a predicate, i.e., $M \in \mathbb{F}_{q}^{\ell \times r}$. For example, some predicate with 4 AND and 5 OR gates as well as 10 variables may be expressed by a $10 \times 5$ matrix, and a predicate with 49 AND and 50 OR gates as well as 100 variables may be expressed by a $100 \times 50$ matrix (see the appendix of [15]). $\lambda$ is the security parameter (e.g., 128).

Table 1. Comparison with the Existing ABS Schemes

\begin{tabular}{|c||c||c|c||c|}
\hline & MPR08 [18] & $\begin{array}{c}\text { MPR10 [19] } \\
\text { (Boneh-Boyen } \\
\text { based) }\end{array}$ & $\begin{array}{c}\text { MPR10 [19] } \\
\text { (Waters } \\
\text { based) }\end{array}$ & Proposed \\
\hline \hline $\begin{array}{c}\text { Signature size } \\
\text { (\# of group elts) }\end{array}$ & $\ell+r+2$ & $51 \ell+2 r+18 \lambda \ell \ell$ & $\begin{array}{c}36 \ell+2 r \\
+9 \lambda+12\end{array}$ & $7 \ell+11$ \\
\hline Model & $\begin{array}{c}\text { generic group } \\
\text { model }\end{array}$ & $\begin{array}{c}\text { standard } \\
\text { model }\end{array}$ & $\begin{array}{c}\text { standard } \\
\text { model }\end{array}$ & $\begin{array}{c}\text { standard } \\
\text { model }\end{array}$ \\
\hline Security & full & full & full & full \\
\hline Assumptions & CR hash & $\begin{array}{c}q \text {-SDH and } \\
\text { DLIN }\end{array}$ & DLIN & $\begin{array}{c}\text { DLIN and } \\
\text { CR hash }\end{array}$ \\
\hline $\begin{array}{c}\text { Sig. size example 1 } \\
(\ell=10, r=5, \\
\lambda=128)\end{array}$ & 17 & monotone & monotone & non-monotone \\
\hline $\begin{array}{c}\text { Sig. size example } 2 \\
(\ell=100, r=50, \\
\lambda=128)\end{array}$ & 152 & 23560 & 1534 & 81 \\
\hline
\end{tabular}




\section{Multi-Authority ABS (MA-ABS)}

\subsection{Definitions and Security of MA-ABS}

We follow the model and security definitions of MA-ABS in [18, 19].

Definition 10 (Multi-Authority ABS : MA-ABS). A multi-authority ABS scheme consists of the following algorithms/protocols.

TSetup. This is a randomized algorithm. The signature trustee runs algorithm $\operatorname{TSetup}\left(1^{\lambda}\right)$ which outputs trustee public key tpk and trustee secret key tsk.

UserReg. This is a randomized algorithm. When a user with user id uid registers with the signature trustee, the trustee runs UserReg(tpk, tsk, uid) which outputs public user-token token $_{\text {uid }}$. The trustee gives token uid $_{\text {to }}$ the user.

ASetup. This is a randomized algorithm. Attribute authority $t(1 \leq t \leq d)$ who wishes to issue attributes runs ASetup(tpk) which outputs attribute-authority

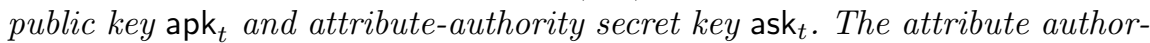
ity, $t$, publishes apk ${ }_{t}$ and stores ask . $_{t}$.

AttrGen. This is a randomized algorithm. When attribute authority $t$ issues user uid a secret key associated with attribute $x_{t}$, first it obtains (from the user) her user-token token $_{\text {uid }}$, and runs token verification algorithm TokenVerify(tpk, uid, token $\left.{ }_{\text {uid }}\right)$. If the token is verified, then it runs AttrGen(tpk, $t$, ask $_{t}$, token $_{\text {uid, }}$, $x_{t}$ ) that outputs attribute secret key usk ${ }_{t}$. The attribute authority gives usk ${ }_{t}$ to the user.

Sig. This is a randomized algorithm. A user signs message $m$ with claim-predicate (access structure) $\mathbb{S}:=(M, \rho)$, only if there is a set of attributes $\Gamma$ such that $\mathbb{S}$ accepts $\Gamma$, the user has obtained a set of keys $\left\{\right.$ usk $_{t} \mid\left(t, x_{t}\right) \in$ $\Gamma\}$ from the attribute authorities. Then signature $\sigma$ can be generated using $\operatorname{Sig}\left(\mathrm{tpk}\right.$, token $_{\text {uid }},\left\{\right.$ apk $_{t}$, usk $\left.\left._{t} \mid\left(t, x_{t}\right) \in \Gamma\right\}, m, \mathbb{S}\right)$, where usk $_{t} \stackrel{\mathrm{R}}{\leftarrow} \operatorname{AttrGen}(\mathrm{tpk}$, $t$, ask ${ }_{t}$, token $\left._{\text {uid }}, x_{t}\right)$.

Ver. To verify signature $\sigma$ on message $m$ with claim-predicate (access structure) $\mathbb{S}$, a user runs $\operatorname{Ver}\left(\mathrm{tpk},\left\{\mathrm{apk}_{t}\right\}, m, \mathbb{S}, \sigma\right)$ which outputs boolean value accept $:=$ 1 or reject $:=0$.

The definition of perfect privacy for the multi-authority (MA) ABS is essentially the same as that of the single-authority (SA) ABS (Definition 8). The major difference of the unforgeability of MA-ABS and SA-ABS is that adversary $\mathcal{A}$ can corrupt an arbitrary subset of attribute authorities provided that adversary $\mathcal{A}$ cannot make a trivial forgery attack. These definitions are given in the full version of this paper.

\subsection{Construction}

The key idea of our construction of MA-ABS scheme is to share $G_{\text {uid }}:=\delta G_{1}$ as well as $G_{0}$ and $G_{1}$ among attribute authorities to generate $\delta \boldsymbol{b}_{t, i}^{*}$ by each authority $t$. Hence, $G_{0}$ and $G_{1}$ are included in tpk and $G_{\text {uid }}:=\delta G_{1}$ is shared with attribute authorities through the user's token token ${ }_{\text {uid }}$. 
For matrix $X:=\left(\chi_{i, j}\right)_{i, j=1, \ldots, N} \in \mathbb{F}_{q}^{N \times N}$ and element $\boldsymbol{v}$ in $N$-dimensional $\mathbb{V}, X(\boldsymbol{v})$ denotes $\sum_{i=1, j=1}^{N, N} \chi_{i, j} \phi_{i, j}(\boldsymbol{v})$ using canonical maps $\left\{\phi_{i, j}\right\}$ (Definition 2). Similarly, for matrix $\left(\vartheta_{i, j}\right):=\left(X^{-1}\right)^{\mathrm{T}},\left(X^{-1}\right)^{\mathrm{T}}(\boldsymbol{v}):=\sum_{i=1, j=1}^{N, N} \vartheta_{i, j} \phi_{i, j}(\boldsymbol{v})$. It holds that $e\left(X(\boldsymbol{x}),\left(X^{-1}\right)^{\mathrm{T}}(\boldsymbol{y})\right)=e(\boldsymbol{x}, \boldsymbol{y})$ for any $\boldsymbol{x}, \boldsymbol{y} \in \mathbb{V}$.

Moreover, (GSIG $, \mathrm{S}, \mathrm{V})$ is a (conventional) unforgeable signature scheme.

$$
\begin{aligned}
& \operatorname{TSetup}\left(1^{\lambda}\right): \quad \operatorname{param}_{\mathbb{G}}:=\left(q, \mathbb{G}, \mathbb{G}_{T}, G, e\right) \stackrel{\mathrm{R}}{\leftarrow} \mathcal{G}_{\mathrm{bpg}}\left(1^{\lambda}\right), \\
& \text { hk } \stackrel{\mathrm{R}}{\leftarrow} \mathrm{KH}_{\lambda}, \quad(\text { verk, sigk }) \stackrel{\mathrm{R}}{\leftarrow} \mathrm{G}_{\mathrm{SIG}}\left(1^{\lambda}\right) \quad N_{0}:=4, N_{d+1}:=7, \quad \kappa, \xi \stackrel{\mathrm{U}}{\longleftarrow} \mathbb{F}_{q}^{\times}, \\
& \text {for } t=0, d+1, \quad \operatorname{param}_{\mathbb{V}_{t}}:=\left(q, \mathbb{V}_{t}, \mathbb{G}_{T}, \mathbb{A}_{t}, e\right):=\mathcal{G}_{\mathrm{dpvs}}\left(1^{\lambda}, N_{t}, \operatorname{param}_{\mathbb{G}}\right) \text {, } \\
& X_{t}:=\left(\chi_{t, i, j}\right)_{i, j} \stackrel{\cup}{\leftarrow} G L\left(N_{t}, \mathbb{F}_{q}\right),\left(\vartheta_{t, i, j}\right)_{i, j}:=\left(X_{t}^{-1}\right)^{\mathrm{T}}, \\
& \boldsymbol{b}_{t, i}:=\kappa\left(\chi_{t, i, 1}, \ldots, \chi_{t, i, N_{t}}\right)_{\mathbb{A}_{t}}, \mathbb{B}_{t}:=\left(\boldsymbol{b}_{t, 1}, \ldots, \boldsymbol{b}_{t, N_{t}}\right) \text {, } \\
& \boldsymbol{b}_{t, i}^{*}:=\xi\left(\vartheta_{t, i, 1}, \ldots, \vartheta_{t, i, N_{t}}\right)_{\mathbb{A}_{t}}, \mathbb{B}_{t}^{*}:=\left(\boldsymbol{b}_{t, 1}^{*}, \ldots, \boldsymbol{b}_{t, N_{t}}^{*}\right), \\
& G_{0}:=\kappa G, \quad G_{1}:=\xi G, \quad g_{T}:=e(G, G)^{\kappa \xi} \text {, } \\
& \widehat{\mathbb{B}}_{0}:=\left(\boldsymbol{b}_{0,1}, \boldsymbol{b}_{0,4}\right), \widehat{\mathbb{B}}_{d+1}:=\left(\boldsymbol{b}_{d+1,1}, \boldsymbol{b}_{d+1,2}, \boldsymbol{b}_{d+1,7}\right), \\
& \widehat{\mathbb{B}}_{d+1}^{*}:=\left(\boldsymbol{b}_{d+1,1}^{*}, \boldsymbol{b}_{d+1,2}^{*}, \boldsymbol{b}_{d+1,5}^{*}, \boldsymbol{b}_{d+1,6}^{*}\right) \text {, } \\
& \text { tsk }:=\left(\boldsymbol{b}_{0,1}^{*}, \text { sigk }\right) \text {, } \\
& \text { tpk }:=\left(1^{\lambda}, \text { hk, }\left\{\text { param }_{\mathbb{V}_{t}}, \widehat{\mathbb{B}}_{t}\right\}_{t=0, d+1}, \boldsymbol{b}_{0,3}^{*}, \widehat{\mathbb{B}}_{d+1}^{*}, g_{T}, G_{0}, G_{1}, \text { verk }\right), \\
& \text { return (tsk, tpk). }
\end{aligned}
$$

UserReg(tpk, tsk, uid) $: \delta \stackrel{U}{\leftarrow} \mathbb{F}_{q}^{\times}, \quad \varphi_{0}, \varphi_{d+1,1, \iota}, \varphi_{d+1,2, \iota} \stackrel{\cup}{\longleftarrow} \mathbb{F}_{q}, \quad G_{\text {uid }}:=\delta G_{1}$,

$$
\begin{aligned}
& \boldsymbol{k}_{0}^{*}:=\left(\delta, 0, \varphi_{0}, 0\right)_{\mathbb{B}_{0}^{*}}, \\
& \boldsymbol{k}_{d+1,1}^{*}:=\left(\delta(1,0), \quad 0,0, \quad \varphi_{d+1,1,1}, \varphi_{d+1,1,2}, \quad 0\right)_{\mathbb{B}_{d+1}^{*}}, \\
& \boldsymbol{k}_{d+1,2}^{*}:=\left(\delta(0,1), \quad 0,0, \varphi_{d+1,2,1}, \varphi_{d+1,2,2}, 0\right)_{\mathbb{B}_{d+1}^{*}}, \\
& \text { usk } \left._{0}:=\left(\boldsymbol{k}_{0}^{*}, \boldsymbol{k}_{d+1,1}^{*}, \boldsymbol{k}_{d+1,2}^{*}\right), \quad \sigma_{\text {uid }}:=\mathrm{S}\left(\text { sigk, (uid, } G_{\text {uid }}\right)\right) \text {, } \\
& \text { return } \text { token }_{\text {uid }}:=\left(\text { uid, } G_{\text {uid }}, \sigma_{\text {uid }}, \text { usk }_{0}\right) \text {. }
\end{aligned}
$$

ASetup(tpk) : $\boldsymbol{u}_{j, i}:=\left(0^{i-1}, G_{j}, 0^{7-i}\right)$ for $j=0,1 ; i=1, . ., 7, X_{t} \longleftarrow G L\left(7, \mathbb{F}_{q}\right)$,

$$
\begin{aligned}
& \mathbb{B}_{t}:=\left(\boldsymbol{b}_{t, i}\right)_{i=1, \ldots, 7}:=\left(X_{t}\left(\boldsymbol{u}_{0,1}\right), \ldots, X_{t}\left(\boldsymbol{u}_{0,7}\right)\right), \\
& \mathbb{B}_{t}^{*}:=\left(\boldsymbol{b}_{t, i}^{*}\right)_{i=1, \ldots, 7}:=\left(\left(X_{t}^{-1}\right)^{\mathrm{T}}\left(\boldsymbol{u}_{1,1}\right), \ldots,\left(X_{t}^{-1}\right)^{\mathrm{T}}\left(\boldsymbol{u}_{1,7}\right)\right), \\
& \widehat{\mathbb{B}}_{t}:=\left(\boldsymbol{b}_{t, 1}, \boldsymbol{b}_{t, 2}, \boldsymbol{b}_{t, 7}\right), \quad \widehat{\mathbb{B}}_{t}^{*}:=\left(\boldsymbol{b}_{t, 1}^{*}, \boldsymbol{b}_{t, 2}^{*}, \boldsymbol{b}_{t, 5}^{*}, \boldsymbol{b}_{t, 6}^{*}\right),
\end{aligned}
$$$$
\text { return }\left(\text { ask }_{t}:=X_{t}, \text { apk }_{t}:=\left(\widehat{\mathbb{B}}_{t}, \widehat{\mathbb{B}}_{t}^{*}\right)\right) \text {. }
$$

TokenVerify(tpk, uid, token uid $)$ holds iff $\mathrm{V}\left(\right.$ verk, $\left(\right.$ uid, $\left.\left.G_{\text {uid }}\right), \sigma_{\text {uid }}\right)=1$.

$\operatorname{AttrGen}\left(\operatorname{tpk}, t\right.$, ask $_{t}$, token $\left._{\text {uid }}, x_{t} \in \mathbb{F}_{q}\right): \varphi_{t, 1}, \varphi_{t, 2} \longleftarrow \mathbb{F}_{q}$, $\boldsymbol{k}_{t}^{*}:=\left(X_{t}^{-1}\right)^{\mathrm{T}}\left(\left(G_{\text {uid }}, x_{t} G_{\text {uid }}, 0,0, \varphi_{t, 1} G_{1}, \varphi_{t, 2} G_{1}, 0\right)\right)$, that is, $\boldsymbol{k}_{t}^{*}=\left(\delta, \delta x_{t}, 0,0, \varphi_{t, 1}, \varphi_{t, 2}, 0\right)_{\mathbb{B}_{t}^{*}}$, return $\mathrm{usk}_{t}:=\boldsymbol{k}_{t}^{*}$. 
Sig(tpk, token uid $_{\text {, }}\left\{\right.$ apk $_{t}$, usk $_{t} \stackrel{\mathrm{R}}{\leftarrow} \operatorname{AttrGen}\left(\mathrm{tpk}, t\right.$, ask $_{t}$, token $\left.\left._{\text {uid }}, x_{t}\right) \mid\left(t, x_{t}\right) \in \Gamma\right\}$, $m, \mathbb{S}:=(M, \rho))$ and $\operatorname{Ver}\left(\mathrm{tpk},\left\{\mathrm{apk}_{t}\right\}_{t=1, \ldots, d}, m, \mathbb{S}:=(M, \rho), \overrightarrow{\boldsymbol{s}}^{*}\right)$ are essentially the same as those in Section 4.2 .

\subsection{Security}

Theorem 3. The proposed $M A-A B S$ scheme is perfectly private.

Theorem 4. The proposed $M A-A B S$ scheme is unforgeable (adaptive-predicate unforgeable) under the DLIN assumption and the existence of collision resistant hash functions.

For any adversary $\mathcal{A}$, there exist probabilistic machines $\mathcal{E}_{1}, \mathcal{E}_{2, h}^{+}, \mathcal{E}_{2, h+1} \quad(h=$ $\left.0, \ldots, \nu_{1}-1\right), \mathcal{E}_{3, h}, \mathcal{E}_{4, h} \quad\left(h=1, \ldots, \nu_{2}\right)$, whose running times are essentially the same as that of $\mathcal{A}$, such that for any security parameter $\lambda$,

$$
\begin{aligned}
\operatorname{Adv}_{\mathcal{A}}^{\mathrm{MA}-\mathrm{ABS}, \mathrm{UF}}(\lambda) \leq \operatorname{Adv}_{\mathcal{E}_{1}}^{\mathrm{DLIN}}(\lambda)+\sum_{h=0}^{\nu_{1}-1}\left(\operatorname{Adv}_{\mathcal{E}_{2, h}^{+}}^{\mathrm{DLIN}}(\lambda)+\operatorname{Adv}_{\mathcal{E}_{2, h+1}}^{\mathrm{DLIN}}(\lambda)\right) \\
+\sum_{h=1}^{\nu_{2}}\left(\operatorname{Adv}_{\mathcal{E}_{3, h}}^{\mathrm{DLIN}}(\lambda)+\operatorname{Adv}_{\mathcal{E}_{4, h}, \mathrm{CR}}^{\mathrm{H}, \lambda)}+\epsilon,\right.
\end{aligned}
$$

where $\nu_{1}$ is the maximum number of $\mathcal{A}$ 's UserReg queries, $\nu_{2}$ is the maximum number of $\mathcal{A}$ 's AltSig queries, and $\epsilon:=\left((2 d+16) \nu_{1}+18 \nu_{2}+2 d+18\right) / q$.

The proofs of Theorems 3 and 4 are given in the full version of this paper.

\section{References}

1. Beimel, A.: Secure schemes for secret sharing and key distribution. PhD Thesis, Israel Institute of Technology, Technion, Haifa, Israel (1996)

2. Belenkiy, M., Camenisch, J., Chase, M., Kohlweiss, M., Lysyanskaya, A., Shacham, H.: Randomizable proofs and delegatable anonymous credentials. In: Halevi, S. (ed.) CRYPTO 2009. LNCS, vol. 5677, pp. 108-125. Springer, Heidelberg (2009)

3. Belenkiy, M., Chase, M., Kohlweiss, M., Lysyanskaya, A.: P-signatures and noninteractive anonymous credentials. In: Canetti, R. (ed.) TCC 2008. LNCS, vol. 4948, pp. 356-374. Springer, Heidelberg (2008)

4. Boyen, X.: Mesh signatures. In: Naor, M. (ed.) EUROCRYPT 2007. LNCS, vol. 4515, pp. 210-227. Springer, Heidelberg (2007)

5. Camenisch, J., Groß, T.: Efficient attributes for anonymous credentials. In: CCS 2008, pp. 345-356. ACM, New York (2008)

6. Camenisch, J., Lysyanskaya, A.: An efficient system for non-transferable anonymous credentials with optional anonymity revocation. In: Pfitzmann, B. (ed.) EUROCRYPT 2001. LNCS, vol. 2045, pp. 93-118. Springer, Heidelberg (2001)

7. Camenisch, J., Lysyanskaya, A.: Signature schemes and anonymous credentials from bilinear maps. In: Franklin, M.K. (ed.) CRYPTO 2004. LNCS, vol. 3152, pp. 56-72. Springer, Heidelberg (2004)

8. Chaum, D.: Security without identification: Transaction systems to make big brother obsolete. CACM 28(10), 1030-1044 (1985)

9. Chaum, D., van Heyst, E.: Group signatures. In: Davies, D.W. (ed.) EUROCRYPT 1991. LNCS, vol. 547, pp. 257-265. Springer, Heidelberg (1991) 
10. Groth, J., Sahai, A.: Efficient non-interactive proof systems for bilinear groups. In: Smart, N.P. (ed.) EUROCRYPT 2008. LNCS, vol. 4965, pp. 415-432. Springer, Heidelberg (2008)

11. Guo, S., Zeng, Y.: Attribute-based signature scheme. In: ISA 2008, pp. 509-511. IEEE, Los Alamitos (2008)

12. Khader, D.: Attribute based group signatures, ePrint, IACR, http://eprint.iacr.org/2007/159

13. Khader, D.: Attribute based group signature with revocation. ePrint, IACR, http://eprint.iacr.org/2007/241

14. Lewko, A., Okamoto, T., Sahai, A., Takashima, K., Waters, B.: Fully Secure Functional Encryption: Attribute-Based Encryption and (Hierarchical) Inner Product Encryption. In: Gilbert, H. (ed.) EUROCRYPT 2010. LNCS, vol. 6110, pp. 62-91. Springer, Heidelberg (2010)

15. Lewko, A.B., Waters, B.: Decentralizing attribute-based encryption. ePrint, IACR, http://eprint.iacr.org/2010/351

16. Li, J., Au, M.H., Susilo, W., Xie, D., Ren, K.: Attribute-based signature and its application. In: ASIACCS 2010, pp. 60-69. ACM, New York (2010)

17. Li, J., Kim, K.: Attribute-based ring signatures. ePrint, IACR, http://eprint.iacr.org/2008/394

18. Maji, H., Prabhakaran, M., Rosulek, M.: Attribute-based signatures: Achieving attribute-privacy and collusion-resistance. ePrint, IACR, http://eprint.iacr.org/2008/328

19. Maji, H., Prabhakaran, M., Rosulek, M.: Attribute-based signatures. To appear in CT-RSA 2011, http://eprint.iacr.org/2010/595

20. Okamoto, T., Takashima, K.: Homomorphic encryption and signatures from vector decomposition. In: Galbraith, S.D., Paterson, K.G. (eds.) Pairing 2008. LNCS, vol. 5209, pp. 57-74. Springer, Heidelberg (2008)

21. Okamoto, T., Takashima, K.: Hierarchical predicate encryption for inner-products. In: Matsui, M. (ed.) ASIACRYPT 2009. LNCS, vol. 5912, pp. 214-231. Springer, Heidelberg (2009)

22. Okamoto, T., Takashima, K.: Fully secure functional encryption with general relations from the decisional linear assumption. In: Rabin, T. (ed.) CRYPTO 2010. LNCS, vol. 6223, pp. 191-208. Springer, Heidelberg (2010), http://eprint.iacr.org/2010/563

23. Rivest, R.L., Shamir, A., Tauman, Y.: How to leak a secret. In: Boyd, C. (ed.) ASIACRYPT 2001. LNCS, vol. 2248, pp. 552-565. Springer, Heidelberg (2001)

24. Shahandashti, S.F., Safavi-Naini, R.: Threshold attribute-based signatures and their application to anonymous credential systems. In: Preneel, B. (ed.) AFRICACRYPT 2009. LNCS, vol. 5580, pp. 198-216. Springer, Heidelberg (2009)

25. Shamir, A.: Identity-based cryptosystems and signature schemes. In: Blakely, G.R., Chaum, D. (eds.) CRYPTO 1984. LNCS, vol. 196, pp. 47-53. Springer, Heidelberg (1985)

26. Waters, B.: Dual system encryption: Realizing fully secure IBE and HIBE under simple assumptions. In: Halevi, S. (ed.) CRYPTO 2009. LNCS, vol. 5677, pp. 619 636. Springer, Heidelberg (2009)

27. Yang, P., Cao, Z., Dong, X.: Fuzzy identity based signature. ePrint, IACR, http://eprint.iacr.org/2008/002 